\title{
Universality in Sherrington-Kirkpatrick’s spin glass model
}

\author{
Philippe Carmona ${ }^{\mathrm{a}}$, Yueyun $\mathrm{Hu}^{\mathrm{b}, *}$ \\ ${ }^{a}$ Laboratoire Jean Leray, UMR 6629, Université de Nantes, 92208, 44322, Nantes cedex 03, France \\ b Département de Mathématiques, Institut Galilée (CNRS UMR-7539), Université Paris XIII, 99, Av. J-B Clément, 93430 Villetaneuse, France
}

Received 19 May 2004; received in revised form 11 March 2005; accepted 4 April 2005

Available online 21 June 2005

\begin{abstract}
It was already clear to Sherrington and Kirkpatrick [Phys. Rev. B 17 (1978) 4384-4403] that the limiting free energy in Sherrington-Kirkpatrick's Spin Glass Model does not depend on the particular distribution of environments. We give here a mathematical proof of this fact.
\end{abstract}

(c) 2005 Elsevier SAS. All rights reserved.

\section{Résumé}

Il est déjà clair pour Sherrington et Kirkpatrick [Phys. Rev. B 17 (1978) 4384-4403] que l'énergie libre de leur modèle de verres de spins ne dépend pas de la loi particulière de l'environnement. Nous donnons ici une démonstration rigoureuse de ce résultat.

(c) 2005 Elsevier SAS. All rights reserved.

\section{Introduction}

The physical system is an $N$-spin configuration $\sigma=\left(\sigma_{1}, \ldots, \sigma_{N}\right) \in\{-1,1\}^{N}$. Each configuration $\sigma$ is given a Boltzmann weight $\mathrm{e}^{\beta H_{N}(\sigma)+h \sum_{i=1}^{N} \sigma_{i}}$ where $\beta=1 / T>0$ is the inverse of the temperature, $h$ is the intensity of the magnetic interaction, $H_{N}(\sigma)$ is the random Hamiltonian

$$
H_{N}(\sigma)=H_{N}(\sigma, \xi)=\frac{1}{\sqrt{N}} \sum_{1 \leqslant i, j \leqslant N} \xi_{i j} \sigma_{i} \sigma_{j}
$$

\footnotetext{
* Corresponding author.

E-mail addresses: philippe.carmona@math.univ-nantes.fr (P. Carmona), yueyun@ math.univ-paris13.fr (Y. Hu).
} 
and $\left(\xi_{i j}\right)_{1 \leqslant i, j \leqslant N}$ is a family of i.i.d. random variables with the same law as $\xi$, admitting order three moments, which we normalize:

$$
\mathbf{E}[\xi]=0, \quad \mathbf{E}\left[\xi^{2}\right]=1, \quad \mathbf{E}\left[|\xi|^{3}\right]<+\infty .
$$

The object of interest is the random Gibbs measure

$$
\langle f(\sigma)\rangle=\frac{1}{Z_{N}} 2^{-N} \sum_{\sigma} f(\sigma) \mathrm{e}^{\beta H_{N}(\sigma, \xi)+h \sum_{i=1}^{N} \sigma_{i}},
$$

and in particular the partition function

$$
Z_{N}=Z_{N}(\beta, \xi)=2^{-N} \sum_{\sigma} \mathrm{e}^{\beta H_{N}(\sigma, \xi)+h \sum_{i=1}^{N} \sigma_{i}}
$$

We shall denote by $g=\left(g_{i j}\right)_{1 \leqslant i, j \leqslant N}$ an environment of i.i.d Gaussian standard random variables $(\mathcal{N}(0,1))$.

Recently, F. Guerra and F.L. Toninelli [1,2] gave a rigorous proof, at the mathematical level, of the convergence of free energy to a deterministic limit, in a Gaussian environment,

$$
\frac{1}{N} \log Z_{N}(\beta, g) \rightarrow \alpha_{\infty}(\beta) \quad \text { a.s. and in average. }
$$

Talagrand [5] then proved that one can replace the Gaussian environment by a Bernoulli environment $\eta_{i j}$, $\mathbf{P}\left(\eta_{i j}= \pm 1\right)=\frac{1}{2}$, and obtain the same limit: $\alpha_{\infty}(\beta)$. We shall generalize this result.

Theorem 1. Assume the environment $\left(\xi_{i j}\right)$ satisfies (1). Then,

$$
\frac{1}{N} \log Z_{N}(\beta, \xi) \rightarrow \alpha_{\infty}(\beta) \text { a.s. and in average. }
$$

Furthermore, the averages $\alpha_{N}(\beta, \xi) \stackrel{\text { def }}{=} \frac{1}{N} \mathbf{E}\left[\log Z_{N}(\beta, \xi)\right]$ satisfy

$$
\left|\alpha_{N}(\beta, \xi)-\alpha_{N}(\beta, g)\right| \leqslant 9 \mathbf{E}\left[|\xi|^{3}\right] \frac{\beta^{3}}{\sqrt{N}} .
$$

Therefore the limiting free energy $\alpha_{\infty}(\beta)$ does not depend on the particular distribution of the environment, as soon as it is constituted of iid random variables satisfying (1), hence the Universality in the title of this note. The independence of the particular disorder was already clear to Sherrington and Kirkpatrick [4] even if they had no mathematical proof of this fact. We mention that Guerra and Toninelli [2] provided a proof when the environment is symmetric and has a finite fourth moment.

Notice eventually that $\alpha_{\infty}(\beta)$ can be determined in a Gaussian framework where Talagrand [6] recently proved that it is the solution of G. Parisi's variational formula.

The universality property can be mechanically extended to the ground states, that is the supremum of the families of random variables:

$$
S_{N}(\xi)=\sup _{\sigma} \sum_{1 \leqslant i, j \leqslant N} \sigma_{i} \sigma_{j} \xi_{i j}=\sqrt{N} \lim _{\beta \rightarrow+\infty} \frac{1}{\beta} \log Z_{N}(\beta, \xi) .
$$

F. Guerra and F.L. Toninelli [1,2] proved that $N^{-3 / 2} S_{N}(g)$ (for the Gaussian environment $g$ ) converges a.s. and in average to a deterministic limit $e_{\infty}$. Here is the general case:

Theorem 2. Assume the environment $\left(\xi_{i j}\right)$ satisfies (1). Then,

$$
N^{-3 / 2} S_{N}(\xi) \rightarrow e_{\infty} \quad \text { a.s. and in average. }
$$


Furthermore, the averages satisfy, for a universal constant $C>0$,

$$
N^{-3 / 2}\left|\mathbf{E}\left[S_{N}(\xi)\right]-\mathbf{E}\left[S_{N}(g)\right]\right| \leqslant C\left(1+\mathbf{E}\left[|\xi|^{3}\right]\right) N^{-1 / 6}
$$

Remark 3. We do not need the random variables $\xi_{i j}$ to share the same distribution. They only need to be independent, to satisfy (1) and such that $\sup _{i j} \mathbf{E}\left[\left|\xi_{i j}\right|^{3}\right]<+\infty$. Then the same conclusions in Theorems 1 and 2 hold.

The proofs of Theorems 1 and 2, given in Section 3, are inspired from F. Guerra's interpolation technique: Comparing an approximative formula of integration by parts for a general variable $\xi$ with the exact formula for a Gaussian variable $g$, we can control the error term and then obtain the comparison of free energies. This will be done in Section 2 in a general framework. Finally, we generalize in Section 4 Talagrand [5]'s result on Bernoulli variables to a wide class of variables.

\section{Comparison of free energies}

Let us begin with an integration by parts formula:

Lemma 4. Let $\xi$ be a real random variable such that $\mathbf{E}\left[|\xi|^{3}\right]<+\infty$ and $\mathbf{E}[\xi]=0$. Let $F: \mathbb{R} \rightarrow \mathbb{R}$ be twice continuously differentiable with $\left\|F^{\prime \prime}\right\|_{\infty}=\sup _{x \in \mathbb{R}}\left|F^{\prime \prime}(x)\right|<+\infty$. Then

$$
\left|\mathbf{E}[\xi F(\xi)]-\mathbf{E}\left[\xi^{2}\right] \mathbf{E}\left[F^{\prime}(\xi)\right]\right| \leqslant \frac{3}{2}\left\|F^{\prime \prime}\right\|_{\infty} \mathbf{E}\left[|\xi|^{3}\right] .
$$

Proof. Observe first, that by Taylor's formula,

$$
\begin{aligned}
& \left|F(\xi)-F(0)-\xi F^{\prime}(0)\right| \leqslant \frac{\xi^{2}}{2}\left\|F^{\prime \prime}\right\|_{\infty}, \\
& \left|F^{\prime}(\xi)-F^{\prime}(0)\right| \leqslant|\xi|\left\|F^{\prime \prime}\right\|_{\infty} .
\end{aligned}
$$

Therefore,

$$
\begin{aligned}
\left|\mathbf{E}[\xi F(\xi)]-\mathbf{E}\left[\xi^{2}\right] \mathbf{E}\left[F^{\prime}(\xi)\right]\right| & =\left|\mathbf{E}[\xi F(\xi)]-\mathbf{E}\left[\xi^{2}\right] \mathbf{E}\left[F^{\prime}(\xi)\right]-F(0) \mathbf{E}[\xi]\right| \\
& =\left|\mathbf{E}\left[\xi\left(F(\xi)-F(0)-\xi F^{\prime}(0)\right)\right]-\mathbf{E}\left[\xi^{2}\right] \mathbf{E}\left[F^{\prime}(0)-F^{\prime}(\xi)\right]\right| \\
& \leqslant\left\|F^{\prime \prime}\right\|_{\infty}\left(\frac{1}{2} \mathbf{E}\left[|\xi|^{3}\right]+\mathbf{E}[|\xi|] \mathbf{E}\left[\xi^{2}\right]\right) \\
& \leqslant\left\|F^{\prime \prime}\right\|_{\infty}\left(\frac{1}{2} \mathbf{E}\left[|\xi|^{3}\right]+\mathbf{E}\left[|\xi|^{3}\right]^{1 / 3} \mathbf{E}\left[|\xi|^{3}\right]^{2 / 3}\right) \\
& \leqslant \frac{3}{2}\left\|F^{\prime \prime}\right\|_{\infty} \mathbf{E}\left[|\xi|^{3}\right] .
\end{aligned}
$$

In the general framework, $X=\left(X_{1}, \ldots, X_{d}\right)$ is a random vector defined on a probability space $(\Omega, \mathcal{F}, \mathbb{P})$ such that for any $i:\left|X_{i}\right| \leqslant 1$. The environment is an i.i.d family of random variables $\left(\xi_{1}, \ldots, \xi_{d}\right)$ defined on $\left(\Omega^{(\xi)}, \mathcal{F}^{(\xi)}, \mathbf{P}\right)$, distributed as a fixed random variable $\xi$ satisfying $(1)$. The Gibbs measure, partition function and averaged free energy are thus

$$
\begin{aligned}
\langle f(X)\rangle & =\frac{1}{Z(\beta, \xi)} \mathbb{E}\left[f(X) \mathrm{e}^{\beta \sum_{i=1}^{d} X_{i} \xi_{i}}\right], \\
Z(\beta, \xi) & =\mathbb{E}\left[\mathrm{e}^{\beta \sum_{i=1}^{d} X_{i} \xi_{i}}\right], \quad \alpha(\beta, \xi)=\mathbf{E}[\log Z(\beta, \xi)] .
\end{aligned}
$$


Observe that to define $\alpha(\beta, \xi)$ we do not need to assume exponential moments for the random variable $\xi$, since $|\log Z(\beta, \xi)| \leqslant|\beta| \sum_{i=1}^{d}\left|\xi_{i}\right|$. We now approximate the derivative of the averaged free energy:

\section{Lemma 5.}

$$
\frac{\partial \alpha(\beta, \xi)}{\partial \beta}=\beta \mathbf{E}\left[\sum_{i=1}^{d}\left(\left\langle X_{i}^{2}\right\rangle-\left\langle X_{i}\right\rangle^{2}\right)\right]+9 d \mathbf{E}\left[|\xi|^{3}\right] \mathrm{O}\left(\beta^{2}\right),
$$

where $\left|\mathrm{O}\left(\beta^{2}\right)\right| \leqslant \beta^{2}$.

Remark 6. In a Gaussian random environment, the integration by parts formula is an exact formula, therefore the remainder $9 d \times \mathbf{E}\left[|\xi|^{3}\right] \mathrm{O}\left(\beta^{2}\right)$ vanishes.

Proof. We have

$$
\frac{\partial \alpha(\beta, \xi)}{\partial \beta}=\mathbf{E}\left[\frac{1}{Z(\beta, \xi)} \mathbb{E}\left[\sum_{i=1}^{d} X_{i} \xi_{i} \mathrm{e}^{\beta \sum_{i=1}^{d} X_{i} \xi_{i}}\right]\right]=\mathbf{E}\left[\sum_{i=1}^{d} \xi_{i} F_{i}\left(\xi_{i}\right)\right],
$$

with $F_{i}(z)=\left(\mathbb{E}\left[X_{i} \mathrm{e}^{\beta X_{i} z+\psi_{i}(X)}\right]\right) /\left(\mathbb{E}\left[\mathrm{e}^{\beta X_{i} z+\psi_{i}(X)}\right]\right)$ and $\psi_{i}(X)=\beta \sum_{j \neq i} X_{j} \xi_{j}$ independent of $\xi_{i}$.

If we define $\langle H\rangle^{(z)}=\left(\mathbb{E}\left[H \mathrm{e}^{\beta X_{i} z+\psi_{i}(X)}\right]\right) /\left(\mathbb{E}\left[\mathrm{e}^{\beta X_{i} z+\psi_{i}(X)}\right]\right)$, then

$$
\frac{\partial}{\partial z}\langle H\rangle^{(z)}=\beta\left(\left\langle H X_{i}\right\rangle^{(z)}-\langle H\rangle^{(z)}\left\langle X_{i}\right\rangle^{(z)}\right)
$$

Hence,

$$
\begin{aligned}
& F_{i}(z)=\left\langle X_{i}\right\rangle^{(z)}, \quad F_{i}^{\prime}(z)=\beta\left(\left\langle X_{i}^{2}\right\rangle^{(z)}-\left(\left\langle X_{i}\right\rangle^{(z)}\right)^{2}\right), \\
& F_{i}^{\prime \prime}(z)=\beta^{2}\left[\left\langle X_{i}^{3}\right\rangle^{(z)}-3\left\langle X_{i}^{2}\right\rangle^{(z)}\left\langle X_{i}\right\rangle^{(z)}+2\left(\left\langle X_{i}\right\rangle^{(z)}\right)^{3}\right] .
\end{aligned}
$$

Since $\left|X_{i}\right| \leqslant 1$, we have $\left\|F_{i}^{\prime \prime}\right\|_{\infty} \leqslant 6 \beta^{2}, 0 \leqslant F_{i}^{\prime}(z) \leqslant \beta$ and

$$
F_{i}\left(\xi_{i}\right)=\left\langle X_{i}\right\rangle, \quad F_{i}^{\prime}\left(\xi_{i}\right)=\beta\left(\left\langle X_{i}^{2}\right\rangle-\left\langle X_{i}\right\rangle^{2}\right) .
$$

We infer from Lemma 4 that since $\mathbf{E}\left[\xi^{2}\right]=1$,

$$
\mathbf{E}\left[\left\langle X_{i}\right\rangle \xi_{i}\right]=\mathbf{E}\left[\xi_{i} F_{i}\left(\xi_{i}\right)\right]=\beta \mathbf{E}\left[\left\langle X_{i}^{2}\right\rangle-\left\langle X_{i}\right\rangle^{2}\right]+9 \mathbf{E}\left[|\xi|^{3}\right] \mathrm{O}\left(\beta^{2}\right),
$$

with $\left|\mathrm{O}\left(\beta^{2}\right)\right| \leqslant \beta^{2}$. Therefore,

$$
\frac{\partial \alpha(\beta, \xi)}{\partial \beta}=\beta \mathbf{E}\left[\sum_{i=1}^{d}\left(\left\langle X_{i}^{2}\right\rangle-\left\langle X_{i}\right\rangle^{2}\right)\right]+9 d \mathbf{E}\left[|\xi|^{3}\right] \times \mathrm{O}\left(\beta^{2}\right) .
$$

The next step is the comparison of the averaged free energies for the environments $\xi$ and $g$ (standard normal).

Proposition 7. For any $\beta \in \mathbb{R}$,

$$
|\alpha(\beta, \xi)-\alpha(\beta, g)| \leqslant 9 d \mathbf{E}\left[|\xi|^{3}\right]|\beta|^{3} .
$$

Proof. The interpolation technique of F. Guerra relies on the introduction of a two parameter Hamiltonian:

$$
Z(t, x)=\mathbb{E}\left[\mathrm{e}^{\sqrt{t} \sum_{i=1}^{d} X_{i} g_{i}+\sqrt{x} \sum_{i=1}^{d} X_{i} \xi_{i}}\right]
$$

and averaged free energy $\alpha(t, x)=\mathbf{E}[\log Z(t, x)]$ where the environments $g$ and $\xi$ are assumed to be independent of each other, $g$ being standard normal. By Lemma 5, 


$$
\begin{aligned}
& \frac{\partial}{\partial t} \alpha=\frac{1}{2} \mathbf{E}\left[\sum_{i=1}^{d}\left\langle X_{i}^{2}\right\rangle-\left\langle X_{i}\right\rangle^{2}\right], \\
& \frac{\partial}{\partial x} \alpha=\frac{1}{2} \mathbf{E}\left[\sum_{i=1}^{d}\left\langle X_{i}^{2}\right\rangle-\left\langle X_{i}\right\rangle^{2}\right]+9 d \mathbf{E}\left[|\xi|^{3}\right] \mathrm{O}(\sqrt{x}),
\end{aligned}
$$

with $|\mathrm{O}(\sqrt{x})| \leqslant \sqrt{x}$. We follow the path $x(s)=t_{0}-s, 0 \leqslant s \leqslant t_{0}$. Then,

$$
\left|\frac{\mathrm{d}}{\mathrm{d} s} \alpha\left(s, t_{0}-s\right)\right| \leqslant 9 d \mathbf{E}\left[|\xi|^{3}\right] \sqrt{t_{0}},
$$

and thus, integrating on $\left[0, t_{0}\right]$

$$
\left|\alpha\left(0, t_{0}\right)-\alpha\left(t_{0}, 0\right)\right| \leqslant 9 d \mathbf{E}\left[|\xi|^{3}\right] t_{0}^{3 / 2} .
$$

This is the desired result for $\beta>0$ (take $\beta=\sqrt{t_{0}}$ ). For negative $\beta$, we consider the environment $-\xi$ instead.

We shall now estimate the fluctuations of free energy, the environment is still constructed with i.i.d random variables $\left(\xi_{1}, \ldots, \xi_{d}\right)$ satisfying (1).

Lemma 8. There exists some universal constant $c>0$ such that

$$
\mathbf{E}\left[|\log Z(\beta, \xi)-\alpha(\beta, \xi)|^{3}\right] \leqslant c \mathbf{E}\left[|\xi|^{3}\right] \beta^{3} d^{3 / 2} .
$$

Consequently, we have

$$
\mathbf{E}\left[\left|\sup _{\left(X_{i}\right)} \sum_{i=1}^{d} X_{i} \xi_{i}-\mathbf{E}\left(\sup _{\left(X_{i}\right)} \sum_{i=1}^{d} X_{i} \xi_{i}\right)\right|^{3}\right] \leqslant c \mathbf{E}\left[|\xi|^{3}\right] d^{3 / 2} .
$$

Proof. It is standard to use the martingale decomposition argument (cf. [3]). Let $\mathcal{F}_{k}=\sigma\left\{\xi_{1}, \ldots, \xi_{k}\right\}, k \geqslant 1$, be the natural filtration generated by $\left(\xi_{k}\right)$. Consider the sequence of martingale difference

$$
\Delta_{j}:=\mathbf{E}\left[\log Z(\beta, \xi) \mid \mathcal{F}_{j}\right]-\mathbf{E}\left[\log Z(\beta, \xi) \mid \mathcal{F}_{j-1}\right], \quad 1 \leqslant j \leqslant d,
$$

with $\mathcal{F}_{0}$ the trivial $\sigma$-field. Then

$$
\log Z(\beta, \xi)-\alpha(\beta, \xi)=\sum_{j=1}^{d} \Delta_{j}
$$

Burkholder's martingale inequality says that for some universal constant $c^{\prime}>0$,

$$
\mathbf{E}\left|\sum_{j=1}^{d} \Delta_{j}\right|^{3} \leqslant c^{\prime} \mathbf{E}\left(\sum_{j=1}^{d} \Delta_{j}^{2}\right)^{3 / 2} .
$$

To estimate $\Delta_{j}$, we define $Z^{(j)}:=\mathbb{E}\left[\mathrm{e}^{\beta \sum_{i=1, i \neq j}^{d} X_{i} \xi_{i}}\right]$ and an auxiliary random probability measure $\mathbb{Q}^{(j)}$ by

$$
\mathbb{Q}^{(j)}\left(F\left(X_{1}, \ldots, X_{d}\right)\right):=\frac{1}{Z^{(j)}} \mathbb{E}\left[F\left(X_{1}, \ldots, X_{d}\right) \mathrm{e}^{\beta \sum_{i=1, i \neq j}^{d} X_{i} \xi_{i}}\right], \quad \forall F(\cdot) \geqslant 0 .
$$

Then

$$
Z(\beta, \xi)=Z^{(j)} \mathbb{Q}^{(j)}\left(\mathrm{e}^{\beta X_{j} \xi_{j}}\right) .
$$


Since $Z^{(j)}$ is independent of $\xi_{j}, \log Z^{(j)}$ has the same conditional expectation with respect to $\mathcal{F}_{j}$ as to $\mathcal{F}_{j-1}$. It follows that

$$
\Delta_{j}=\mathbf{E}\left(\log \mathbb{Q}^{(j)}\left(\mathrm{e}^{\beta X_{j} \xi_{j}}\right) \mid \mathcal{F}_{j}\right)-\mathbf{E}\left(\log \mathbb{Q}^{(j)}\left(\mathrm{e}^{\beta X_{j} \xi_{j}}\right) \mid \mathcal{F}_{j-1}\right) .
$$

Using the fact that $\left|X_{j}\right| \leqslant 1$, we get $\left|\log \mathbb{Q}^{(j)}\left(\mathrm{e}^{\beta X_{j} \xi_{j}}\right)\right| \leqslant \beta\left|\xi_{j}\right|$. This implies that

$$
\left|\Delta_{j}\right| \leqslant \beta\left(\left|\xi_{j}\right|+\mathbf{E}\left|\xi_{j}\right|\right)
$$

It follows that

$$
\begin{aligned}
\mathbf{E}|\log Z(\beta, \xi)-\alpha(\beta, \xi)|^{3} & \leqslant c^{\prime} \mathbf{E}\left(\sum_{j=1}^{d} \Delta_{j}^{2}\right)^{3 / 2} \leqslant c^{\prime} \beta^{3} \mathbf{E}\left(\sum_{j=1}^{d}\left(\left|\xi_{j}\right|+\mathbf{E}\left|\xi_{j}\right|\right)^{2}\right)^{3 / 2} \\
& \leqslant c^{\prime} \beta^{3} \sqrt{d} \sum_{j=1}^{d} \mathbf{E}\left(\left|\xi_{j}\right|+\mathbf{E}\left|\xi_{j}\right|\right)^{3} \leqslant c \mathbf{E}|\xi|^{3} \beta^{3} d^{3 / 2}
\end{aligned}
$$

where we used the convexity of the function $x \rightarrow x^{3 / 2}$ in the third inequality. Finally, considering $\frac{1}{\beta} \log Z(\beta, \xi)$ and letting $\beta \rightarrow \infty$, we obtain the second estimate and end the proof.

\section{Application to Sherrington-Kirkpatrick's model of spin glass}

Observe that

$$
Z_{N}(\beta, \xi)=2^{-N} \sum_{\sigma} \mathrm{e}^{\frac{\beta}{\sqrt{N}} H_{N}(\sigma, \xi)+h \sum_{i} \sigma_{i}}=\mathbb{E}\left[\mathrm{e}^{(\beta / \sqrt{N}) H_{N}(\tau, \xi)+h \sum_{i} \tau_{i}}\right],
$$

where $\left(\tau_{i}\right)_{1 \leqslant i \leqslant N}$ are i.i.d with distribution $\mathbb{P}\left(\tau_{i}=\mp 1\right)=\frac{1}{2}$. We get rid of the magnetic field by introducing tilted laws:

$$
\mathbb{P}\left(\tilde{\tau}_{i}= \pm 1\right)=\frac{(1 / 2) \mathrm{e}^{ \pm h}}{\cosh (h)}, \quad \text { so that } \quad \mathbb{E}\left[f\left(\tilde{\tau}_{i}\right)\right]=\frac{\mathbb{E}\left[f\left(\tau_{i}\right) \mathrm{e}^{h \tau_{i}}\right]}{\mathbb{E}\left[\mathrm{e}^{h \tau_{i}}\right]} .
$$

With these notations we have

$$
Z_{N}(\beta, \xi)=\cosh (h)^{N} \mathbb{E}\left[\mathrm{e}^{(\beta / \sqrt{N}) H_{N}(\tilde{\tau}, \xi)}\right] .
$$

\subsection{Convergence of free energy: Proof of Theorem 1}

Applying Proposition 7 to $X_{i j}=\tilde{\tau}_{i} \tilde{\tau}_{j}, \beta \rightarrow \beta / \sqrt{N}$ and $d=N^{2}$ yields

$$
\left|\alpha_{N}(\beta, \xi)-\alpha_{N}(\beta, g)\right|=\frac{1}{N}\left|\alpha\left(\frac{\beta}{\sqrt{N}}, \xi\right)-\alpha\left(\frac{\beta}{\sqrt{N}}, g\right)\right| \leqslant \frac{1}{N} 9 N^{2} \mathbf{E}\left[|\xi|^{3}\right]\left(\frac{\beta}{\sqrt{N}}\right)^{3}=9 \mathbf{E}\left[|\xi|^{3}\right] \frac{\beta^{3}}{\sqrt{N}} .
$$

Furthermore, the fluctuations can be controlled by Lemma 8:

$$
\mathbf{E}\left[\left|\frac{1}{N} \log Z_{N}(\beta, \xi)-\alpha_{N}(\beta, \xi)\right|^{3}\right] \leqslant c \mathbf{E}|\xi|^{3} \beta^{3} N^{-3 / 2},
$$

this gives the a.s. convergence by Borel-Cantelli's Lemma. 


\subsection{Convergence of ground state: Proof of Theorem 2}

We have, restricting the sum to a configuration yielding a maximum Hamiltonian to get the lower bound,

$$
\mathrm{e}^{(\beta / \sqrt{N}) S_{N}(\xi)} \geqslant Z_{N}(\beta, \xi)=2^{-N} \sum_{\sigma} \mathrm{e}^{(\beta / \sqrt{N}) H_{N}(\sigma, \xi)} \geqslant 2^{-N} \mathrm{e}^{(\beta / \sqrt{N}) S_{N}(\xi)} .
$$

Therefore,

$$
\frac{1}{\sqrt{N}} \mathbf{E}\left[S_{N}(\xi)\right] \geqslant \frac{1}{\beta} N \alpha_{N}(\beta, \xi) \geqslant \frac{1}{\sqrt{N}} \mathbf{E}\left[S_{N}(\xi)\right]-\frac{N \log 2}{\beta} .
$$

Combining with inequality (2) yields, by taking $\beta=N^{1 / 6}$

$$
\begin{aligned}
\frac{1}{N^{3 / 2}}\left|\mathbf{E}\left[S_{N}(g)\right]-\mathbf{E}\left[S_{N}(\xi)\right]\right| & \leqslant \frac{2 \log 2}{\beta}+\frac{1}{\beta}\left|\alpha_{N}(\beta, \xi)-\alpha_{N}(\beta, g)\right| \leqslant \frac{2 \log 2}{\beta}+C \mathbf{E}\left[|\xi|^{3}\right] \frac{\beta^{2}}{\sqrt{N}} \\
& \leqslant C^{\prime}\left(1+\mathbf{E}\left[|\xi|^{3}\right]\right) N^{-1 / 6} .
\end{aligned}
$$

The almost sure convergence follows in the same way from the control of fluctuations and Borel-Cantelli's Lemma.

\section{Some extensions and generalizations}

\subsection{The p-spin model of spin glasses}

The partition function is

$$
Z_{N}(\beta, \xi)=2^{-N} \sum_{\sigma} \mathrm{e}^{\left(\beta / \sqrt{N^{p-1}}\right) H_{N}(\sigma, \xi)+h \sum_{i} \sigma_{i}}=\mathbb{E}\left[\mathrm{e}^{\left(\beta / \sqrt{N^{p-1}}\right) H_{N}(\tau, \xi)+h \sum_{i} \tau_{i}}\right],
$$

where $\left(\tau_{i}\right)_{1 \leqslant i \leqslant N}$ are i.i.d with distribution $\mathbb{P}\left(\tau_{i}=\mp 1\right)=\frac{1}{2}$ (we get rid of the magnetic field by introducing tilted laws so we assume, without loss in generality, that $h=0$ ).

The Hamiltonian is

$$
H_{N}(\sigma, \xi)=\sum_{1 \leqslant i_{1}, \ldots, i_{p} \leqslant N} \sigma_{i_{1}} \cdots \sigma_{i_{p}} \xi_{i_{1} \ldots i_{p}}
$$

where $\xi_{i_{1} \ldots i_{p}}$ is an iid family of random variables with common distribution satisfying (1).

Applying Proposition 7 to $X_{i_{1} \ldots i_{p}}=\tilde{\tau}_{i_{1}} \ldots \tilde{\tau}_{i_{p}}, \beta \rightarrow \frac{\beta}{\sqrt{N^{p-1}}}$ and $d=N^{p}$ yields

$$
\left|\alpha_{N}(\beta, \xi)-\alpha_{N}(\beta, g)\right| \leqslant 9 \mathbf{E}\left[|\xi|^{3}\right] \frac{\beta^{3}}{N^{(p-1) / 2}} .
$$

\subsection{Integration by parts and comparison of free energies}

The more information we get on the random media, the more precise our comparison results can be. In particular, the more Gaussian the environment looks like, the closer the free energy is to the Gaussian free energy. For example, we shall assume here that the random variable $\xi$ satisfies

$$
\mathbf{E}\left[|\xi|^{4}\right]<+\infty, \quad \mathbf{E}[\xi]=\mathbf{E}\left[\xi^{3}\right]=0, \quad \mathbf{E}\left[\xi^{2}\right]=1
$$

A typical variable in this class is the Bernoulli $\mathbf{P}[\eta= \pm 1]=\frac{1}{2}$. We get the approximate integration by parts formula 
Lemma 9. Assume that the real random variable $\xi$ satisfies (3) and that the function $F: \mathbb{R} \rightarrow \mathbb{R}$ is of class $C^{3}$ with bounded third derivative $\left\|F^{(3)}\right\|_{\infty}<+\infty$. Then,

$$
\left|\mathbf{E}[\xi F(\xi)]-\mathbf{E}\left[\xi^{2}\right] \mathbf{E}\left[F^{\prime}(\xi)\right]\right| \leqslant\left\|F^{(3)}\right\|_{\infty} \mathbf{E}\left[\xi^{4}\right] .
$$

Proof. This is again Taylor's formula:

$$
\begin{aligned}
& F(\xi)=F(0)+\xi F^{\prime}(0)+\frac{1}{2} \xi^{2} F^{\prime \prime}(0)+\mathrm{O}\left(\left|\xi^{3}\right|\left\|F^{(3)}\right\|_{\infty}\right), \\
& F^{\prime}(\xi)=F^{\prime}(0)+\xi F^{\prime \prime}(0)+\mathrm{O}\left(\xi^{2}\left\|F^{(3)}\right\|_{\infty}\right) .
\end{aligned}
$$

Repeating, mutatis mutandis, the proof of Proposition 7 we obtain

Proposition 10. There exists a constant $C>0$ such that for any environment $\xi$ satisfying (3), and for a Gaussian environment $g$,

$$
|\alpha(\beta, \xi)-\alpha(\beta, g)| \leqslant C \mathbf{E}\left[\xi^{4}\right] d \beta^{4} .
$$

In the framework of Sherrington-Kirkpatrick model of spin glass, this yields

$$
\left|\alpha_{N}(\beta, \xi)-\alpha_{N}(\beta, g)\right| \leqslant C \mathbf{E}\left[\xi^{4}\right] \frac{\beta^{4}}{N} .
$$

The ground state comparison is now

$$
N^{-3 / 2}\left|\mathbf{E}\left[S_{N}(\xi)\right]-\mathbf{E}\left[S_{N}(g)\right]\right| \leqslant C\left(1+\mathbf{E}\left[|\xi|^{4}\right]\right) N^{-1 / 4} .
$$

This is of the same order as Talagrand's result (Corollary 1.2 of [5]) established for Bernoulli random variables.

\section{Acknowledgements}

We gratefully acknowledge fruitful conversations with Francesco Guerra, held during his stay at Université de Nantes as "Professeur Invité" in 2003 and 2004. We also thank Professor Leonid Pastur for helpful remarks.

\section{References}

[1] F. Guerra, Broken replica symmetry bounds in the mean field spin glass model, Comm. Math. Phys. 233 (1) (2003) 1-12.

[2] F. Guerra, L.T. Toninelli, The thermodynamic limit in mean field spin glass models, Comm. Math. Phys. 230 (1) (2002) 71-79.

[3] L.A. Pastur, M.V. Shcherbina, Absence of self-averaging of the order parameter in the Sherrington-Kirkpatrick model, J. Statist. Phys. 62 (1-2) (1991) 1-19.

[4] D. Sherrington, S. Kirkpatrick, Infinite-ranged model of spin-glasses, Phys. Rev. B 17 (1978) 4384-4403.

[5] M. Talagrand, Gaussian averages, Bernoulli averages, and Gibbs' measures, Random Struct. Algorithms 21 (3-4) (2002) 197-204.

[6] M. Talagrand, The Parisi formula, Ann. of Math., in press. 